\section{Lethal tracheal dissolution during treatment for thyroid lymphoma}

\author{
Anton Melnyk, Nancy J Graham, \\ Donna Weber, Garrett L Walsh
}

\begin{abstract}
Background - A case of primary thyroid T cell lymphoma leading to lethal tracheal perforation during chemotherapy is described.
\end{abstract}

(Thorax 1995;50:1120-1121)

Keywords: thyroid lymphoma, tracheal perforation.

Fatal perforation of the stomach or small bowel is a well recognised complication following chemotherapy for gastrointestinal lymphoma. ${ }^{1}$ We present a case of fatal necrosis and perforation of the trachea in a patient treated for a locally invasive thyroid lymphoma. To the best of our knowledge, this complication has not previously been reported.

\section{Case report}

A 74 year old man with emphysema presented with a six month history of sore throat, cough, hoarseness, and mild dysphagia. Additional systemic complaints included general malaise, fever, night sweats, and a $9 \mathrm{~kg}$ weight loss.

Physical examination revealed mild inspiratory stridor, a $5 \mathrm{~cm} \times 6 \mathrm{~cm}$ mass in the left lobe of the thyroid gland, an $8 \mathrm{~cm} \times 6 \mathrm{~cm}$ subcutaneous right axillary mass, and two smaller subcutaneous truncal lesions. The patient had no palpable lymphadenopathy. Indirect laryngoscopy showed a mass involving the left pyriform sinus and oedema of the left aryepiglottic fold.

An intravenous contrast enhanced computed Department of A Melnyk D Weber

Department of Diagnostic Imaging N J Graham

Department of Thoracic and Cardiovascular Surgery G L Walsh Texas MD Anderson Cancer Center, Houston, Texas 77030, USA

Reprint requests to Dr G L Walsh.

Received 21 April 1994 Accepted for publication 28 July 1995 showed a large heterogeneous thyroid mass with marked irrregularity of the adjacent anterior and lateral tracheal walls due to tumour invasion (fig 1). The tumour and associated tracheal wall irregularity extended caudally to $4 \mathrm{~cm}$ above the tracheal carina. No mediastinal lymphadenopathy or pulmonary metastases were identified. A CT scan of the abdomen and pelvis and bone marrow analysis were normal

Intravenous dexamethasone was administered to alleviate airway compromise following fine needle aspiration of the thyroid mass, which showed that the tumour was an intermediate grade $\mathrm{T}$ cell lymphoma. The patient's stridor improved markedly in response to steroid therapy while staging investigations were completed. The lymphoma was staged as
The University of

IVB, with two sites of bulky disease in the thyroid and skin.

Chemotherapy consisting of cisplatin, doxorubicin, and methylprednisolone was initiated. On the second day of this regimen the patient developed subcutaneous emphysema and dyspnoea following a paroxysm of coughing. Increasing respiratory distress necessitated endotracheal intubation with only transient improvement of symptoms. The patient's condition again deteriorated, with a dramatic worsening of subcutaneous emphysema in the neck and chest (fig 2) and difficulty with mechanical ventilation. He was transiently resuscitated from electromechanical dissociation by repositioning of the endotracheal tube.

Bronchoscopic examination revealed necrotic tumour and blood almost completely occluding the upper airway. In an effort to re-establish a patent airway a bedside tracheostomy was performed for a presumed high tracheal perforation. The cartilaginous portion of the trachea was completely necrotic from the cricoid cartilage to $4 \mathrm{~cm}$ above the carina. The remaining distal trachea, which had retracted into the mediastinum, was delivered into the neck incision by an Allis clamp and intubated with a long tracheostomy tube. After a short period of ventilatory and haemodynamic stability, all remaining cartilaginous rings dissolved. The patient died of extensive necrosis of the entire cartilaginous trachea.

\section{Discussion}

Thyroid lymphoma is a rare tumour comprising approximately $2 \%$ of extranodal non-Hodgkin's lymphomas, most of which are of an intermediate histological grade. This tumour most commonly affects women in the sixth decade who present with a rapidly expanding neck mass, often with a history of Hashimoto's thyroiditis. ${ }^{2}$ Many of the masses are larger than $5 \mathrm{~cm}$ at presentation, and almost $60 \%$ have extended beyond the gland at diagnosis. ${ }^{34}$ Symptoms secondary to local invasion are com-

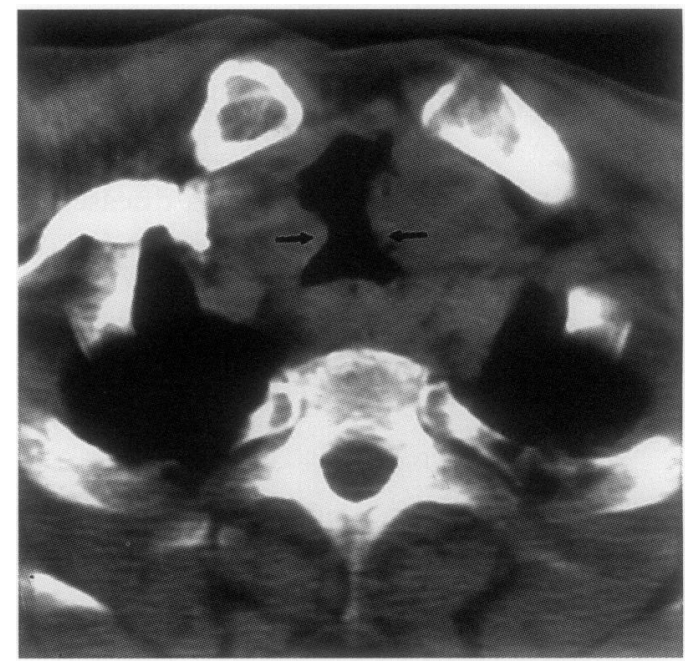

Figure $110 \mathrm{~mm}$ collimation transverse CT scan at the level of the lung apices showing a mass involving the thyroid gland with marked irregularity of the tracheal wall (arrows). 
Figure 2 Portable chest radiograph after intubation showing extensive subcutaneous emphysema of the upper chest and neck. The cervical trachea is markedly distended (arrows).

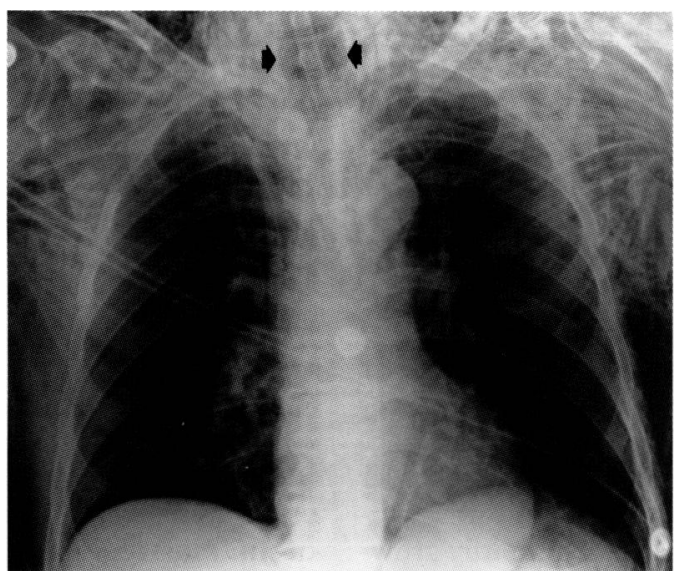

to mention whether the tumour invaded the trachea or larynx. ${ }^{4}$ In one report two patients demonstrated obstructive symptoms and biopsy proven tracheal invasion by thyroid lymphoma; both patients received chemotherapy with resolution of obstruction and without tracheal perforation, and both were alive one and two years later without evidence of disease. $^{5}$

Computed tomographic scanning is the recommended method of assessment of extrathyroid extension of lymphoma. ${ }^{8}$ Early awareness of tracheal involvement by lymphoma should alert the clinician to the remote possibility of tracheal dissolution during treatment of this extremely difficult clinical problem.

mon: hoarseness (9-67\%), dysphagia (9-60\%), and dyspnoea or stridor (9-35\%). ${ }^{5}$ Although the optimal treatment for thyroid lymphoma is uncertain, patients with stage IIE or more advanced disease should probably receive chemotherapy in addition to local treatment (surgery and/or radiotherapy). ${ }^{6}$

Perforation of the gastrointestinal tract at the site of disease in patients with gastrointestinal lymphoma who are receiving chemotherapy has been well described; ${ }^{1}$ a similar perforation has been reported in a patient with thyroid lymphoma metastatic to the small bowel. ${ }^{7}$

Lethal tracheal perforation during chemotherapy has not previously been described in a patient with primary thyroid lymphoma. Most series of patients with thyroid lymphoma fail
1 Randall J, Obeid ML, Blackledge GR. Haemorrhage and perforation of gastrointestinal neoplasms during chemotherapy. Ann $R$ Coll Surg Engl 1986;68:286-9.

2 Shaw JHF, Holden A, Sage M. Thyroid lymphoma. $B r f$ Surg 1989;76:895-7.

3 Tupchong L, Hughes F, Harmer CL. Primary lymphoma of the thyroid: clinical features, prognostic factors, and results of treatment. Int $¥$ Radiat Oncol Biol Phys 1986;12:1813-21.

4 Hamburger JI, Miller JM, Kini SR. Lymphoma of the thyroid. Hamburger JI, Miller JM, Kini SR. Lym
Ann Intern Med 1983;99:685-93.

5 Van Ruiswyk J, Cunningham C, Cereletty J. Obstructive manifestations of thyroid lymphoma. Arch Intern Med 1989, 149:1575-7. lymphomas stages IE and IIE: comparative results for radiotherapy only, combination chemotherapy only, and multimodality treatment. Int $\mathcal{F}$ Radiat Oncol Biol Phys 1986 12:1807-12.

7 McDermott EWM, Cassidy N, Heffernan SJ. Perforation through undiagnosed small bowel involvement in primary 572-3.

8 Takashima S, Morimoto S, Ikezoe J, Arisawa J, Hamada S, Ikeda $\mathrm{H}$, et al. Primary thyroid lymphoma: comparison of CT and US assessment. Radiology 1989;171:439-43.
6 Vigliotti A, Kong JS, Fuller LM, Velasquez WS. Thyroid thyroid lymphoma during chemotherapy. Cancer 1992;69:

\section{Commentary: lymphoma involving the mediastinum - challenges in diagnosis and management}

\section{Jonathan A Ledermann}

Lymphomas are the seventh most common malignancy and the incidence of non-Hodgkin's lymphoma is increasing. Correct diagnosis is important as they are treatable and, in some cases, curable. Lymphomas can involve any organ system and, as thoracic involvement is common, they often present to respiratory physicians. The diagnosis is not always straightforward and treatment is complex. The case reports in this issue of Thorax illustrate some of the diagnostic and therapeutic problems associated with this disease.

Mediastinal tumours in adults are divided fairly equally throughout the mediastinal compartments. Approximately $40 \%$ of tumours are found in the anterosuperior compartment. Thymomas are the most common tumour in this region and lymphomas and carcinoids may involve the thymus. Mediastinal germ cell tumours are located in the anterosuperior compartment and thyroid tumours may involve the upper part of the superior mediastinum. Secondary tumours, particularly bronchogenic carcinoma, should also always be considered. Thymomas are often suspected by their radiological appearance and they may have associated systemic features, most notably myasthenia gravis. They are usually treated by surgical extirpation which allows further detailed study of the pathology and avoids the increased incidence of local recurrence seen if needle biopsy is performed before surgery. For other masses radiologically guided needle biopsy has become commonplace and has reduced the need for operative biopsy. Fine needle aspiration is simple and may be sufficient to diagnose a carcinoma. However, as Robinson et al point out, the diagnosis of lymphoma may be missed unless adequate tissue is removed. This is required for study of the morphology of the tumour which provides prognostic information and assists management. A comprehensive immunohistological examination should be performed in case misleading information is obtained from one immuno-

\footnotetext{
Schoon Medical

London W1P 8BT J A Ledermann

Department of Oncology, University College
} 